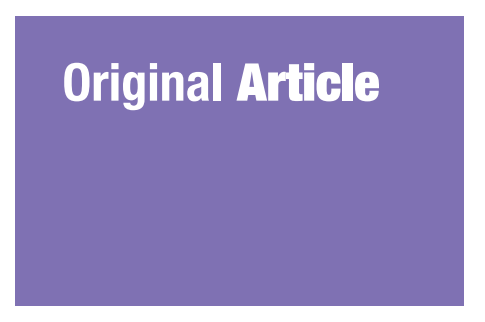

Submitted: 6 Mar 2020

Accepted: 23 May 2020

Online: 30 Jun 2020

\section{Lipid Peroxidation Induces Reactive Astrogliosis by Activating WNT/ $\beta$-Catenin Pathway in Hydrocephalus}

\author{
Wihasto Suryaningtyas ${ }^{1}$, Muhammad Arifin Parenrengl ${ }^{1}$, Abdul \\ Hafid BAJAMAL ${ }^{1}$, Fedik Abdul RANTAM ${ }^{2}$
}
1 Department of Neurosurgery, Faculty of Medicine Universitas Airlangga, Dr. Soetomo General Hospital, Surabaya, Indonesia
2 Department of Veterinary Microbiology, Faculty of Veterinary Medicine and Laboratory for Stem Cell Research, Institute of Tropical Disease, Universitas Airlangga, Surabaya, Indonesia

To cite this article: Suryaningtyas W, Parenrengi MA, Bajamal AH, Rantam FA. Lipid peroxidation induces reactive astrogliosis by activating WNT/ $\beta$-catenin pathway in hydrocephalus. Malays J Med Sci. 2020;27(3):34-42. https:// doi.org/10.21315/mjms2020.27.3.4

To link to this article: https://doi.org/10.21315/mjms2020.27.3.4

\title{
Abstract
}

Background: Hydrocephalus induces mechanical and biochemical changes in neural cells of the brain. Astrogliosis, as the hallmark of cellular changes in white matter, is involved in demyelination process, re-myelination inhibitory effect, and inhibition of axonal elongation and regeneration. The pathophysiology of this process is not well understood. The purpose of the present study is to elucidate the effect of lipid peroxidation product on astrogliosis through WNT/ $\beta$-catenin in kaolin-induced hydrocephalic rats.

Methods: The study used kaolin-induced hydrocephalic rats. Obstructive hydrocephalus was expected to develop within seven days after induction. The hydrocephalus animals were killed at day 7, 14 and 21 after induction. One group of the saline-injected animals was used for shamtreatment.

Results: We demonstrated that the hydrocephalic rats exhibited a high expression of 4-hydroxynonenal (4-HNE) in the periventricular area. The expression of $\beta$-catenin also increased, following the pattern of 4-HNE. Reactive astrocyte, expressed by positive glial fibrillary acidic protein (GFAP), was upregulated in an incremental fashion as well as the microglia.

Conclusion: This work suggests that lipid peroxidation product, 4-HNE, activated the WNT/ $\beta$-catenin pathway, leading to the development of reactive astrocyte and microglia activation in hydrocephalus.

Keywords: hydrocephalus, lipid peroxidation, $W N T / \beta$-catenin signaling, reactive astrocyte, microglia

\section{Introduction}

Hydrocephalus is a multifactorial neurologic disorder that can occur in the congenital or acquired setting. Mechanical forces and metabolic changes overlap each other and play a central role in structural and cellular changes. Cerebral blood flow alteration occurs due to the stretching and compression of the capillary vessels in the periventricular area $(1,2)$. Reduction in cerebral blood flow causes hypoxia, which induces several biochemical cascades including generation of reactive oxygen species (ROS)/reactive nitrate species (RNS), $\mathrm{Ca}^{2+}$ influx and generation of reactive hydroxyl molecules. Cell membrane suffers from lipid peroxidation induced by oxidative stress when reactive species level exceeds the antioxidant enzyme level. Some studies revealed the accumulation of lipid peroxidation products, i.e. malondialdehyde (MDA) and 4-hydroxynonenal (4-HNE). Other cellular changes documented in many hydrocephalus studies were astrocyte reaction 
and microglial activation, together known as astrogliosis. Both these reactions are a normal response of the brain to injury in the acute phase. However, it is attributed to its detrimental effect on structural and functional recovery in a chronic setting in the form of periventricular hypertrophy and proliferation of astrocytes and microglia $(3,4)$. The chronic reaction may lead to glial scar development that impairs axonal regeneration, inhibits remyelination and causes demyelination $(5,6)$. Once the reactive astrocytosis is established, shunt placement only temporarily ceases the process and will rise again later, suggesting that surgical treatment fails to completely ameliorate or reverse this reaction in the long run (7).

$\mathrm{WNT} / \beta$-catenin is a critical conserved signaling pathway involved in the development of multiple organs. It influences the various arrays of the biological process including cell proliferation and differentiation, organ development, tissue homeostasis, stem cell maintenance and pathogenesis of many diseases such as degenerative inflammation and carcinogenesis. Activation of $\mathrm{WNT} / \beta$-catenin depends mainly on the canonical pathway. A previous study revealed its relation to astroglial response in hydrocephalus. Inhibiting $W N T / \beta-$ catenin pathway and its gene product cyclin D-1 alleviated reactive astrogliosis that was shown as decreasing glial fibrillary acidic protein (GFAP) (8). Microglia were also affected by $W N T / \beta-$ catenin in a neurodegenerative study. Specific WNT ligand could increase pro-inflammatory cytokine produced by activated microglia.

The relation between lipid peroxidation and activation of $\mathrm{WNT} / \beta$-catenin in hydrocephalus has not been studied. We hypothesised that lipid peroxidation is the subsequent reaction of oxidative stress-induced activation of $\mathrm{WNT} / \beta$ catenin. This event led to the development of astrogliosis.

\section{Methods}

\section{Animal}

Sprague-Dawley rats aged 10 weeks $(n=24)$ were used for the experiment. They were housed in standard cages and provided with a normal $12 \mathrm{~h}$ dark/light schedule with free access to food and water. The rats in the hydrocephalus group were sacrificed at 7th, 14th and 21st days and the rats in the sham-treated group were sacrificed on the 21st day after injection.

\section{Hydrocephalus Induction and Specimen Preparation}

Hydrocephalus was induced in 18 rats using percutaneous kaolin suspension injection into the cisterna magna as previously described (9). In brief, anesthesia was accomplished with an intravenous injection of ketamine/xylazine $(90 / 10 \mathrm{mg} / \mathrm{kg})$. The occipital area and the lower neck were shaved and prepared with $70 \%$ ethanol and $10 \%$ povidone-iodine. We placed the rat's chest on a $10 \mathrm{~cm}$ thick sponge support so that the neck could be flexed to open the foramen magnum. A slow injection of $0.05 \mathrm{~mL}$ of $20 \%$ kaolin suspension through foramen magnum was employed. All rats were observed until they recovered from anesthesia and then housed in a standard environment. Hydrocephalus was developed within seven days after injection. The clinical diagnosis of hydrocephalus was made by observing its mobility, gait abnormality and the hunched back appearance. The sham-treated rats only received a sterile saline injection at the same place as the hydrocephalus groups. On the designated days, the rats were sacrificed by decapitating the head. The brain was harvested and fixed for $48 \mathrm{~h}$ in $4 \%$ paraformaldehyde at room temperature.

\section{Immunohistochemistry Staining}

The brain was embedded in a paraffin block and sliced in $5 \mu \mathrm{m}$ sections. For immunohistochemistry (IHC) staining, the paraffin sections were stained with mouse monoclonal antibody (Santa Cruz Biotechnology, Texas) for detecting the expressions of 4-HNE (1:300 dilution), Ki-67 (1:500 dilution), GFAP (1:500 dilution) and Iba-1 (1:500 dilution) according to the manufacturer's instructions. Images from slides were viewed at $20 \times$ and $40 \times$ magnifications under Nikon H6ooL microscope, camera DS Fi2 and analysed using NIS Elements Basic Research imaging software (Nikon Corp, Japan). The results were scored using Immuno Reactive Score (IRS) as suggested by Remmele and Stegner (10). 


\section{Statistical Analysis}

Data were presented as mean \pm SD. Statistical analysis for comparison among groups was performed using ANOVA. Statistical significance was considered for a $P$-value of 0.05 or less. The test was performed using statistic software SPSS version 24 (IBM, Armonk, NY, USA). Path analysis using Categorical Regression with optimal scaling was performed to determine the correlation coefficient between variables (beta coefficient).

\section{Results}

\section{Hydrocephalus Increased 4-HNE \\ Expressions}

Immunohistochemical appearance of 4-HNE showed that the lipid peroxidation process was involved in hydrocephalus (Figure 1). The hydrocephalus group demonstrated a significantly higher 4-HNE expression $(P<0.001)$ on day $7(8.97 \pm$ 2.98) and day $21(11.52 \pm 0.43)$ than shamtreated group (3.61 \pm 0.99) (Figure 2). Immunohistochemistry results showed that the periventricular area, hippocampus, external capsule, and striatum had a higher expression of 4-HNE compared to the cortical area. The coefficient correlation between hydrocephalus and 4-HNE (beta coefficient $=-0.878$; $P<0.001)$ showed that hydrocephalus was significantly upregulated at 4-HNE level.

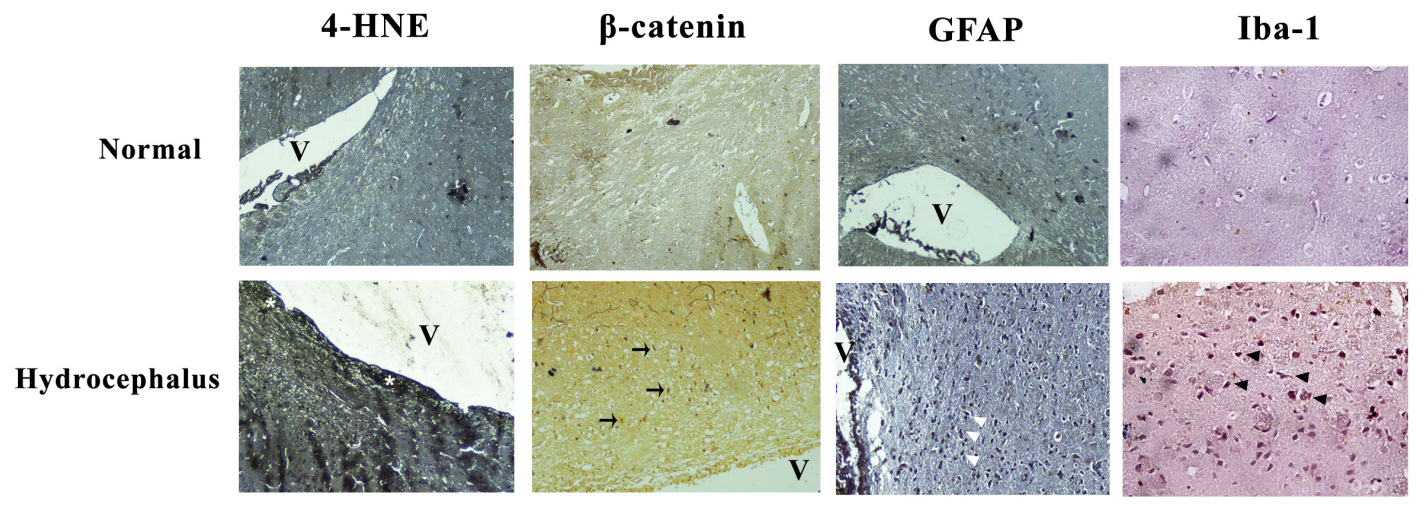

Figure 1. The representative images of 4-HNE, $\beta$-catenin, GFAP and Iba-1 expressions in the periventricular white matter of normal and hydrocephalic rats. Expression of 4-HNE (white asterisk) was prominent in hydrocephalic group, especially in periventricular area. Expression of $\beta$-catenin (black arrow) was higher in hydrocephalic rat than in normal group. GFAP positive cells (white arrowhead) that represented reactive astrocytes were more abundant in hydrocephalus brain compared to the normal brain. The appearance of Iba-1 positive cells (black arrowhead) showed that the microglia was activated in hydrocephalus group. Activated microglia were barely visible in normal group. All images were taken at $40 \times$ magnification except Iba- 1 that was taken at $100 \times$ magnification 

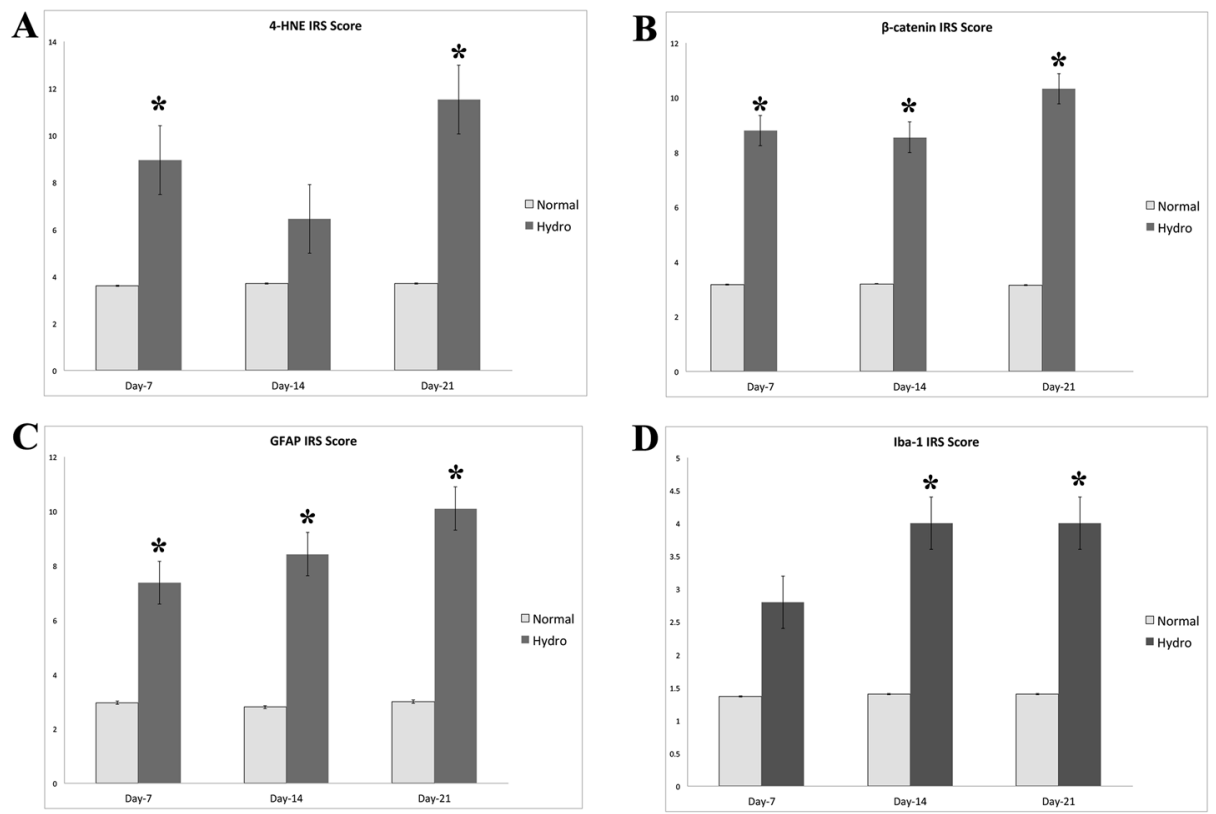

Figure 2. The expressions of (A) 4-HNE, (B) $\beta$-catenin, (C) GFAP and (D) Iba-1. All protein expressions were documented as IRS score. The difference between the hydrocephalic and normal group is considered significant when $P<0.05$ (asterisk)

\section{4-HNE Induced WNT/B-Catenin Activation}

We investigated the WNT/ $\beta$-catenin canonical pathway activation by measuring the cytoplasm $\beta$-catenin level (Figure 1). The immunohistochemistry score of $\beta$-catenin expression was significantly higher in hydrocephalic rats than in the sham-treated group $(P<0.05)$. Its level followed the fluctuated pattern of 4 -HNE. We found $\beta$-catenin score increased in day 7 (8.70 \pm 0.88$)$, slightly declining in day $14(8.43 \pm 0.42)$ and increasing further in day $21(10.41 \pm 0.42)$ (Figure 2). There was no significant difference between day 7 and day 14. The coefficient correlation between 4 - $\mathrm{HNE}$ and $\mathrm{WNT} / \beta$-catenin (beta coefficient $=$ $-0.843 ; P<0.001$ ) showed a significant positive correlation between the 4-HNE levels and WNT/ $\beta$-catenin. The adjusted $\mathrm{R}^{2}$ revealed that $80 \%$ of the WNT/ $\beta$-catenin activator was through lipid peroxidation products. The correlation of increased 4-HNE with WNT/ $\beta$-catenin activation was measured in path analysis.

\section{WNT/B-Catenin Activation Stimulated Reactive Astrocytes}

Reactive astrocyte was considered as the pathological hallmark in hydrocephalus. The hydrocephalus group showed a significantly higher GFAP expression $(P<0.001)$ than in the normal group $(2.96 \pm 0.28)$. In the hydrocephalus group, GFAP expressions increased from day $7(7.47 \pm 0.77)$ to day 21 $(10.21 \pm 0.10)$ (Figure 2). The activation of $\mathrm{WNT} / \beta$-catenin was correlated significantly with astrocyte reactivity as shown by the positive significant correlation (beta coefficient $=-0.987 ; P<0.001)$ between $\mathrm{WNT} / \beta$-catenin and expression of reactive astrocyte.

\section{Microglial Activation in Hydrocephalic Rats}

Increased expression of Iba-1 was found in several areas of the brain, especially at the periventricular area, white matter (external capsule), and striatum after induction of hydrocephalus (Figure 1). The hydrocephalus group had a significantly higher score of Iba-1 at day $14(4.00 \pm 0.90)$ and day $21(4.00 \pm 0.42)$ compared to the normal group $(1.38 \pm 0.60)$ $(P<0.001)$ (Figure 2). The coefficient correlation 
between hydrocephalus and microglial activation (beta coefficient $=-0.754 ; P<0.001$ ) showed that hydrocephalus was significantly activated microglia.

\section{Discussion}

Hydrocephalus induces injury to the brain through mechanical and biochemical alterations. Hydrocephalic rat model exhibited a hunched-back appearance, less mobility, gait abnormality, and balance impairment. Their hind limbs moved in a short wide-based step. The progressive ventricular enlargement causes mechanical stress to the periventricular white matter and the cerebral blood vessels in that area $(2,11)$. The biomechanical injury starts as the blood flow decreases further, triggering the hypoxic-ischemic cascade. The proteinlipid damage and lipid peroxidation at the cellular level occur due to oxidative stress. It produces aldehyde such as malondialdehyde (MDA) and 4-HNE $(12,13)$. In our study, the expression of 4-HNE increased 3 to 4 times in the hydrocephalic animal. Del Bigio et al. (12) also reported similar evidence but to a lesser degree. On day 14 after induction, the expression of 4-HNE declined temporarily and bounced up on day 21 after kaolin induction (Figure 2). This fluctuating pattern of 4-HNE showed a temporary recovered blood flow in two weeks after the induction or cessation of oxidative process in the early period of hydrocephalus development. Correlating the evidence of hydrocephalus with lipid peroxidation product, 4-HNE, captured the evidence of lipid peroxidation process.

The involvement of 4-HNE in modulating downstream signaling has been reported (14). The association of 4 -HNE with WNT/ $\beta$-catenin signaling was reported in diabetic retinopathy (15). Although WNT/ $\beta$-catenin signaling modulates various biological and pathological processes, its association with hydrocephalus has not been established. There is only one study by $\mathrm{Xu}$ et al. that reported the involvement of $\mathrm{WNT} / \beta$-catenin in hydrocephalus (8). Our study showed that the high level of 4 -HNE in hydrocephalic rats induced $\mathrm{WNT} / \beta$-catenin. The upregulated 4 -HNE level increased the activity of $\mathrm{WNT} / \beta$-catenin. The correlation was significantly positive with a correlation factor of 0.8 . It means $80 \%$ of the $\mathrm{WNT} / \beta$-catenin were through the lipid peroxidation product. Its involvement in the signaling pathway, especially in the canonical WNT pathway, was revealed in this study.

WNTs are secreted cysteine-rich glycoproteins ligands that bind to a complex of frizzled (Fz) receptors and low-density lipoprotein receptor-related protein 5 or 6 (LRP5/6) and regulate target genes expression through an intracellular signaling pathway (16). WNT ligands-LRP6 binding dimerises with the Fz receptor, which is the critical step in the activation of the WNT pathway. In the basal state, $\beta$-catenin level, the downstream effector of the canonical $\mathrm{WNT} / \beta$-catenin pathway is kept low by a 'destruction protein complex' containing glycogen synthase kinase$3 \beta$ (GSK $3 \beta)$. The WNT-Fz-LRP $5 / 6$ complex will inhibit the phosphorylation of $\beta$-catenin that results in $\beta$-catenin accumulation. $\beta$-catenin is then translocated into the nucleus and binds with the T-cell factor to regulate the expression of target genes (16). Regarding the mechanism of WNT/ $\beta$-catenin pathway activation by $4-\mathrm{HNE}$, it increases phosphorylation of LRP6, an initial step in WNT/ $\beta$-catenin pathway activation. However, the exact mechanism remains unclear. One possible explanation is that 4-HNE modifies LRP6 or proteasome and inhibits its degradation. This assumption is supported by the report that HNE inhibits LRP6 degradation rather than increasing its production (15).

Activation of $\mathrm{WNT} / \beta$-catenin plays a role in astrogliosis reaction in hydrocephalus. Our study showed a similar result that astrogliosis was correlated with the activation of WNT/ $\beta$ catenin pathway. Currently, there are three theories regarding the origin of reactive astrocytes following brain injury. First, GFAP+ astrocytes come from itself that go through the dedifferentiation process and then divide. Second, it comes from oligodendrocyte progenitors that are found throughout the white matter. Third, it is originated from subventricular zone migrating progenitors. The activation mechanism of reactive astrocyte by $\mathrm{WNT} / \beta$-catenin involving oligodendrocyte progenitor cell (OPC) is supported by evidence that WNT/ $\beta$-catenin signaling occurred in GFAP+ cells and progenitor cells. High $\beta$-catenin tone inhibited oligodendrocyte progenitor maturation into immature oligodendrocyte. On the other hand, high WNT-3a increased GFAP+ cells (17-19). As the number of mature oligodendrocyte falls, the remyelination process will be altered. Inhibition of $\mathrm{WNT} / \beta$-catenin signaling in knockout animals reduced the OPC 
reaction that surrounded the penumbra of the injured area. Suppression of OPC reaction to injury muted the reactions of microglia and astrocytes. The reduction of the GFAP+ reactive astrocyte occurred on $5^{\text {th }}$ day post-injury (20).

Reactive astrocyte has dual appointments based on its appearance period. Its protective action is expected to appear at the early phase as it can eliminate excessive extracellular glutamate and store and supply energy to neighboring cells. Its protective effect will decline after seven days and the neurotoxic phenotype will replace it (21). The hallmark of the neurotoxic state is increased inflammatory chemokine-cytokine, glial scar-forming extracellular matrix and inhibition of synaptogenesis $(22,23)$. WNT/ $\beta$ catenin signaling is also related to pathogenic development of glial scar in the brain as well as in different organs, such as lung fibrosis, liver fibrosis, skin fibrosis, and renal fibrosis (24). Detrimental effects of glial scar include inhibition of axonal sprouting (25), remyelination (26), and axonal regeneration as well as production of an extracellular matrix, chondroitin sulfate proteoglycan (CSPG), that are involved in demyelination process $(27,28)$. Several studies addressed these detrimental effects on neural regeneration and plasticity. Surgical and nonsurgical measures were explored to minimise or inhibit the astrogliosis with varying degree of success $(29,30)$. A surgical approach by shunting resulted in a partial reduction of reactive astrocytosis. The inflammatory phenotype continued to express at the later stage of the chronically shunted animal (31).
Microglia activation in hydrocephalus involved TLR-4 and RAGE as the receptor of Damage associated molecular patterns (DAMPS) and downstream signaling of the nuclear factor- $\kappa \mathrm{B}$ (NF- $\mathrm{kB})$ pathway (32). Activated microglia has a modulatory effect in the development of astrocytosis and determines the property of astrocytes under pathological conditions. The neurotoxic reactive astrocyte phenotype is induced by microglia-derived cytokines. Microglia, through NF- $\kappa \mathrm{B}$ signaling, potentially amplify the inflammatory reaction by upregulating the level of cytokines and chemokines. Interleukin-1 alpha (IL-1 $\alpha$ ) and TNF- $\alpha$ are two classic cytokines that induce the neurotoxic type of reactive astrocyte. This phenotype shows a compromised ability in synaptogenesis, promotion of neuronal survival, and release of neurotoxins, which induce apoptosis in neurons and oligodendroglia (22). Reactive astrocyte secretes chemokines: monocyte chemoattractant protein (MCP)-1/ CCL2 and IFN- $\gamma$ inducible protein (IP)-10/ CXCL10. These chemokines play a role in microglial activation as well as motility. It allows the recruitment of microglia and circulating macrophages to enter the brain parenchyma through a compromised blood-brain barrier. The reactive astrocyte also secreted Lipocalin-2 (Lcn2), which enhances microglial inflammatory activity (33).

Taken together, the results of our study and the latest theories in hydrocephalic pathology revealed the involvement of hypoxia-induced cascade that eventually activates the microgliaastrocyte crosstalk (Figure 3). 


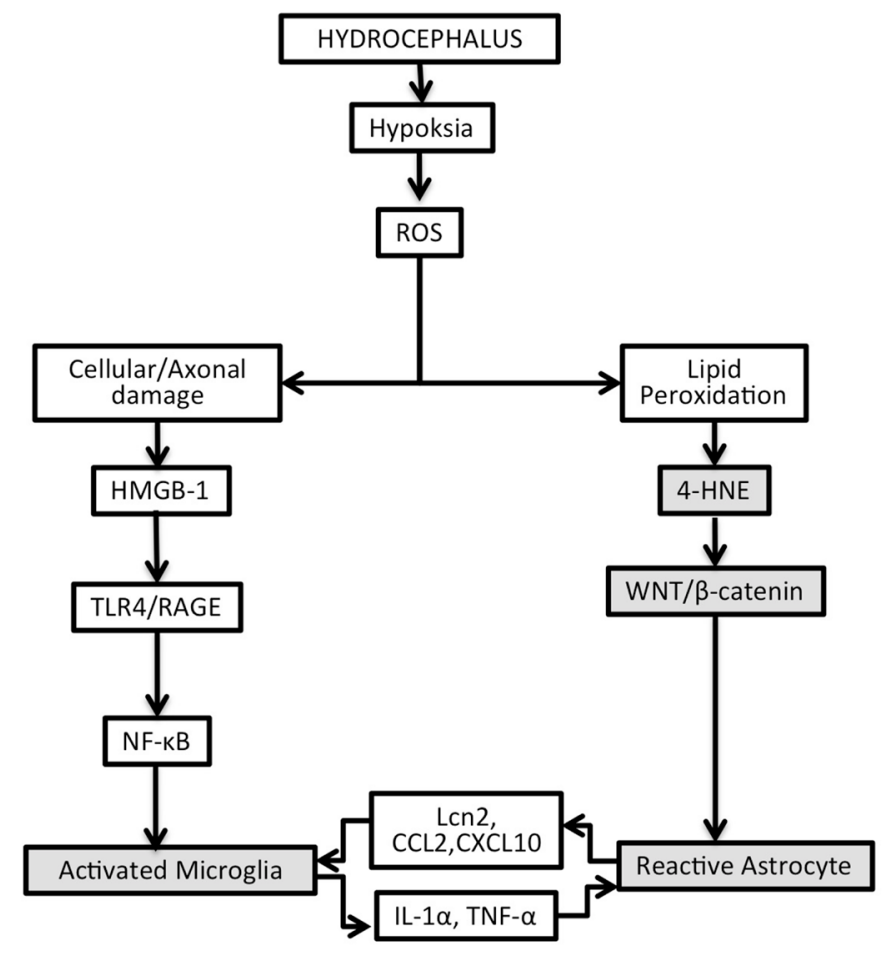

Figure 3. The diagram depicts the pathobiology of astrogliosis as the product of cellular biochemical changes in hydrocephalus

\section{Conclusion}

The hallmarks of cellular changes in hydrocephalus are development of reactive astrocyte and microglial activation. Hypoxiainduced lipid peroxidation activated WNT/ $\beta$ catenin signaling pathway through its aldehyde products, 4-HNE, which in turn upregulated GFAP+ reactive astrocytes and microglial activation.

\section{Acknowledgements}

None.

\section{Ethics of Study}

All procedures involving animals performed in this study were in accordance with the ethical standards of the Institutional Animal Care and Use Committee, Universitas Airlangga, Surabaya, Indonesia.

\section{Conflict of Interest}

None.

\section{Funds}

None.

\section{Correspondence}

Dr Wihasto Suryaningtyas

MD (Universitas Airlangga), PhD (Universitas

Airlangga)

Department of Neurosurgery,

Faculty of Medicine Universitas Airlangga,

Dr. Soetomo General Hospital,

Gedung Pusat Diagnostik Terpadu (GDC)

Lantai 5,

RSUD Dr. Soetomo, Jl. Mayjen Prof Moestopo 6-8,

Surabaya, Indonesia.

Tel: +62 315501325

Fax: +62 315025188

E-mail: wihasto-s@fk.unair.ac.id 


\section{References}

1. Socci DJ, Bjugstad KB, Jones HC, Pattisapu JV, Arendash GW. Evidence that oxidative stress is associated with the pathophysiology of inherited hydrocephalus in the H-Tx rat model. Exp Neurol. 1999;155(1):109-117. https://doi.org/10.1006/ exnr.1998.6969

2. Da Silva MC, Michowicz S, Drake JM, Chumas PD, Tuor UI. Reduced local cerebral blood flow in periventricular white matter in experimental neonatal hydrocephalus-restoration with CSF shunting. $J$ Cereb Blood Flow Metab. 1995;15(6):1057-1065. https://doi.org/10.1038/ jcbfm.1995.132

3. Del Bigio MR. Neuropathology and structural changes in hydrocephalus. Dev Disabil Res Rev. 2010;16(1):16-22. https://doi.org/10.1002/ ddrr.94

4. Deren KE, Packer M, Forsyth J, Milash B, Abdullah OM, Hsu EW, et al. Reactive astrocytosis, microgliosis and inflammation in rats with neonatal hydrocephalus. Exp Neurol. 2010;226(1):110-119. https://doi.org/10.1016/j .expneurol.2010.08.010

5. Sofroniew MV. Molecular dissection of reactive astrogliosis and glial scar formation. Trends Neurosci. 2009;32(12):638-647. https://doi.org/10.1016/j.tins.2009.08.002

6. Silver J. The glial scar is more than just astrocytes. Exp Neurol. 2016;286(2016):147149. https://doi.org/10.1016/j.expneurol.2016.06 .018

7. Eskandari R, Harris CA, McAllister JP. Reactive astrocytosis in feline neonatal hydrocephalus: acute, chronic, and shunt-induced changes. Child's Nerv Syst. 2011;27(12):2067-2076. https://doi.org/10.1007/soo381-011-1552-4

8. $\mathrm{Xu} \mathrm{H}, \mathrm{Xu} \mathrm{B}$, Wang ZX, Tan GW, Shen SH. Inhibition of Wnt/ $\beta$-catenin signal is alleviated reactive gliosis in rats with hydrocephalus.Child's Nerv Syst. 2015;31(2):227-234. https://doi.org/ 10.1007/s00381-014-2613-2

9. Khan $\mathrm{OH}$, Del Bigio MR. Experimental models of hydrocephalus. In: Tatlisumak T, Fisher M, editors. Handbook of experimental neurology: methods and techniques in animal research. Cambridge, UK: Cambridge University Press; 2006. pp. 457-471.
10. Kaemmerer D, Peter L, Lupp A, Schulz S, Sanger Jorg, Baum RP, et al. Comparing of IRS and Her2 as immunohistochemical scoring schemes in gastroenteropancreatic neuroendocrine tumors. Int J Clin Exp Pathol. 2012;5(3):187-194.

11. Momjian S, Owler BK, Czosnyka Z, Czosnyka M, Pena A, Pickard JD. Pattern of white matter regional cerebral blood flow and autoregulation in normal pressure hydrocephalus. Brain. 2004;127(5):965-972. https://doi.org/10.1093/ brain/awh131

12. Bigio MR Del, Khan $\mathrm{OH}$, Lopes S, Juliet PAR. Cerebral white matter oxidation and nitrosylation in young rodents with kaolin-induced hydrocephalus. $J$ Neuropathol Exp Neurol. 2012;71(4):274-288

13. Aras M, Urfali B, Serarslan Y, Ozgur T. Protective effects of minocycline against short-term ischemia-reperfusion injury in rat brain. Pediatr Neurosurg. 2014;49(3):172-178. https://doi.org/ 10.1159/000362202

14. Ayala A, Muñoz MF, Argüelles S. Lipid peroxidation: production, metabolism, and signaling mechanisms of malondialdehyde and 4-hydroxy-2-nonenal. Oxid Med Cell Longev. 2014;2014:1-31. https://doi.org/10.1155/2014/ 360438

15. Zhou T, Zhou KK, Lee K, Gao G, Lyons TJ, Kowluru R, et al. The role of lipid peroxidation products and oxidative stress in activation of the canonical wingless-type MMTV integration site (WNT) pathway in a rat model of diabetic retinopathy. Diabetologia. 2011;54(2):459-468. https://doi.org/10.1007/s00125-010-1943-1

16. Zeng $\mathrm{X}$, Huang $\mathrm{H}$, Tamai $\mathrm{K}$, et al. Initiation of Wnt signaling: control of Wnt coreceptor Lrp6 phosphorylation/activation via frizzled, dishevelled and axin functions. Development. 2007;135(2):367-375. https://doi.org/10.1242/ dev.013540

17. Shimizu T, Kagawa T, Wada T, Muroyama Y, Takada S, Ikenaka K. Wnt signaling controls the timing of oligodendrocyte development in the spinal cord. Dev Biol. 2005;282(2):397-410. https://doi.org/10.1016/j.ydbio.2005.03.020 
18. White BD, Nathe RJ, Maris DO, Nguyen NK, Goodson JM, Moon RT, et al. $\beta$-catenin signaling increases in proliferating NG21 progenitors and astrocytes during post-traumatic gliogenesis in the adult brain. Stem Cells. 2010;28(2):297-307. https://doi.org/10.1002/stem.268

19. Fancy SPJ, Harrington EP, Baranzini SE, Silbereis JC, Shiow LR, Yuen TJ, et al. Parallel states of pathological Wnt signaling in neonatal brain injury and colon cancer. Nat Neurosci. 2014;17(4):506-512. https://doi.org/10.1038/nn .3676

20. Rodriguez JP, Coulter M, Miotke J, Meyer RL, Takemaru K-I, Levine JM. Abrogation of betacatenin signaling in oligodendrocyte precursor cells reduces glial scarring and promotes axon regeneration after CNS injury. $J$ Neurosci. 2014;34(31):10285-10297. https://doi.org/10 $.1523 /$ JNEUROSCI.4915-13.2014

21. Hu X, Li P, Guo Y, Wang H, Leak RK, Chen $\mathrm{S}$, et al. Microglia/macrophage polarization dynamics reveal novel mechanism of injury expansion after focal cerebral ischemia. Stroke. 2012;43(11):3063-3070. https://doi .org/10.1161/STROKEAHA.112.659656

22. Liddelow SA, Guttenplan KA, Clarke LE, Bennett FC, Bohlen CJ, Schirmer L, et al. Neurotoxic reactive astrocytes are induced by activated microglia. Nature. 2017;541(7638):481-487. https://doi.org/10.1038/nature21029

23. Liddelow SA, Barres BA. Reactive astrocytes: production, function, and therapeutic potential. Immunity. 2017;46(6):957-967. https://doi.org /10.1016/j.immuni.2017.06.006

24. Guo Y, Xiao L, Sun L, Liu F. Wnt/ $\beta$-catenin signaling: a promising new target for fibrosis diseases. Physiol Res. 2012;61(4):337-346. https://doi.org/932289 [pii]

25. Siebert JR, Conta Steencken A, Osterhout DJ. Chondroitin sulfate proteoglycans in the nervous system: inhibitors to repair. Biomed Res Int. 2014;2014:1-15. https://doi .org/10.1155/2014/845323
26. Wang $\mathrm{Y}$, Cheng $\mathrm{X}$, He Q, Zheng $\mathrm{Y}$, Kin $\mathrm{DH}$, Whittemore SR, et al. Astrocytes from the contused spinal cord inhibit oligodendrocyte differentiation of adult oligodendrocyte precursor cells by increasing the expression of bone morphogenetic proteins. $J$ Neurosci. 2011;31(16):6053-6058. https://doi.org/10.1523 /JNEUROSCI.5524-09.2011

27. Siebert JR, Osterhout DJ. The inhibitory effects of chondroitin sulfate proteoglycans on oligodendrocytes. $J$ Neurochem. 2011;119(1):176188. https://doi.org/10.1111/j.1471-4159.2011 $.07370 . x$

28. Deng YP, Sun Y, Hu L, Li Z-H, Xu Q-M, Pei Y-L, et al. Chondroitin sulfate proteoglycans impede myelination by oligodendrocytes after perinatal white matter injury. Exp Neurol. 2015;269:213-223. https://doi.org/10.1016/j .expneurol.2015.03.026

29. Del Bigio MR, Di Curzio DL. Nonsurgical therapy for hydrocephalus: a comprehensive and critical review. Fluids Barriers CNS. 2015;13(1):3. https://doi.org/10.1186/s12987-016-0025-2

30. Di Curzio DL, Turner-Brannen E, Del Bigio MR. Oral antioxidant therapy for juvenile rats with kaolin-induced hydrocephalus. Fluids Barriers CNS. 2014;11(1):1-10. https://doi .org/10.1186/2045-8118-11-23

31. Eskandari R, Harris CA, McAllister JP. Reactive astrocytosis in feline neonatal hydrocephalus: acute, chronic, and shunt-induced changes. Child's Nerv Syst. 2011;27(12):2067-2076. https://doi.org/10.1007/soo381-011-1552-4

32. Kettenmann H, Hanisch U-K, Noda M, Verkhratsky A. Physiology of microglia. Physiol Rev. 2011;91(2):461-553. https://doi .org/10.1152/physrev.0o011.2010

33. Jha MK, Lee S, Park DH, Kook H, Park K-G, Lee $\mathrm{I}-\mathrm{K}$, et al. Diverse functional roles of lipocalin-2 in the central nervous system. Neurosci Biobehav Rev. 2015;49:135-156. https://doi.org/10.1016/j .neubiorev.2014.12.006 